\title{
Genome-wide identification and integrated analysis of IncRNAs in rice backcross introgression lines $\left(\mathrm{BC}_{2} \mathrm{~F}_{12}\right)$
}

\author{
Mengdi Li ${ }^{\dagger}$, Aqin $\mathrm{CaO}^{\dagger}$, Ruihua Wang, Zeyu Li, Shaoqing Li and Jianbo Wang ${ }^{*}$
}

\begin{abstract}
Background: Distant hybridization is an important way to create interspecific genetic variation and breed new varieties in rice. A lot of backcross introgression lines (BILs) had been constructed for the scientific issues in rice. However, studies on the critical regulatory factor IncRNA in cultivated rice, wild rice and their BIL progenies were poorly reported.

Results: Here, high-throughput RNA sequencing technology was used to explore the functional characteristics and differences of IncRNAs in $O$. sativa, $O$. longistaminata and their three $\mathrm{BC}_{2} \mathrm{~F}_{12}$ progenies. A total of 1254 IncRNAs were screened out, and the number of differentially expressed IncRNAs between progenies and $O$. sativa were significantly less than that between progenies and O. longistaminata. Some IncRNAs regulated more than one mRNA, and $89.5 \%$ of IncRNAs regulated the expression of target genes through cis-acting. A total of 78 IncRNAs and 271 mRNAs were targeted by 280 miRNAs, and 22 IncRNAs were predicted to be the precursor of 20 microRNAs. Some miRNAs were found to target their own potential precursor IncRNAs. Over $50 \%$ of IncRNAs showed parental expression level dominance (ELD) in all three progenies, and most IncRNAs showed ELD-O. sativa rather than ELD-O. longistaminata. Further analysis showed that InCRNAs might regulate the expression of plant hormone-related genes and the adaptability of $\mathrm{O}$. sativa, $\mathrm{O}$. longistaminata and their progenies.

Conclusions: Taken together, the above results provided valuable clues for elucidating the functional features and expression differences of IncRNAs between O. sativa, O. longistaminata and their BIL progenies, and expanded our understanding about the biological functions of IncRNAs in rice.
\end{abstract}

Keywords: Long non-coding RNA, Backcross introgression lines, Progenies, Transcriptome sequencing, Rice

\section{Background}

Rice (Oryza sativa L.) is one of the staple foods for world population, and its yield is crucial for global food production. With the advantages of moderate genome size and high-quality reference genome, rice is widely regarded as a typical model plant to study the genetic structure and function of monocotyledons. Genus Oryza has evolved into 24 species, consisting of 2 cultivated

\footnotetext{
* Correspondence: jbwang@whu.edu.cn

${ }^{\dagger}$ Mengdi Li and Aqin Cao contributed equally to this work.

State Key Laboratory of Hybrid Rice, College of Life Sciences, Wuhan University, Wuhan 430072, China
}

species (O. sativa and O. glaberrima) and 22 wild species $[1,2]$. Cultivated rice has lost many important useful genes after a long period of artificial selection. However, wild rice, which has experienced harsh natural environment, contains a large number of valuable genes and is a valuable resource in rice breeding [3]. Interspecific distant hybridization between different species with excellent genes and distant genetic relationships is an important way to create genetic variation and breed new varieties, which is also an important driver of genome evolution and speciation [4]. A generally accepted effective strategy to expand the genetic diversity of cultivated

(c) The Author(s). 2020 Open Access This article is licensed under a Creative Commons Attribution 4.0 International License, which permits use, sharing, adaptation, distribution and reproduction in any medium or format, as long as you give appropriate credit to the original author(s) and the source, provide a link to the Creative Commons licence, and indicate if changes were made. The images or other third party material in this article are included in the article's Creative Commons licence, unless indicated otherwise in a credit line to the material. If material is not included in the article's Creative Commons licence and your intended use is not permitted by statutory regulation or exceeds the permitted use, you will need to obtain permission directly from the copyright holder. To view a copy of this licence, visit http://creativecommons.org/licenses/by/4.0/ The Creative Commons Public Domain Dedication waiver (http://creativecommons.org/publicdomain/zero/1.0/) applies to the data made available in this article, unless otherwise stated in a credit line to the data. 
rice is to identify and utilize valuable alleles with agronomic traits from wild rice and introduce them into cultivated rice by crossbreeding backcross $[5,6]$. In the past few decades, a lot of backcross introgression lines (BILs) had been constructed to study scientific issues in rice, such as drought resistance [7, 8] genomic structure [3], hybrid sterility [5] and gene, miRNA and metabolic profiling $[2,9]$. O. longistaminata is a perennial wild rice widely distributed in tropical Africa with strong resistance to biotic and abiotic stresses, strong rhizomes, long anthers and self-incompatibility [10-12].

Long non-coding RNA (lncRNA) refers to RNA with a length of more than $200 \mathrm{bp}$ and no protein coding capacity. LncRNAs are involved in a variety of molecular and genetic mechanisms, including transcriptional level, post-transcriptional level and epigenetic level [13, 14]. LncRNAs are involved in many biological processes, including growth of human tumor cells, plant morphogenesis, biotic stress and abiotic threats [15-17]. The functions of lncRNAs are divided into four categories, including signal, decoy, guide, and scaffold [18]. Specifically, lncRNAs could regulate spatial/temporal expression of genes when they act as signal molecules [19]. LncRNAs could participate in maintaining the stability of gene expression by acting as decoys or target mimics of miRNAs [2022]. LncRNAs could also guide the ribonucleoprotein complex to locate at a specific site to play their guiding role [23]. In addition, lncRNAs could be used as scaffolds to form skeletal complexes with transcription factors, which can regulate the up-/down-stream effector elements and further activate or inhibit the transcription of genes [24]. Moreover, lncRNAs could regulate the expression of protein-coding genes at the transcriptional level through cis-regulation or transregulation. When lncRNA cis-regulates the target gene, it is encoded from the nucleic acid chain in which their target gene was located. On the contrary, the nucleic acid sequence encoding lncRNA was not on the same nucleic acid chain as the target gene coding sequence when lncRNA trans-regulate the target gene. For instance, a lncRNA (COLDAIR) cis-regulated the FLOWERING LOCUS C (FLC) gene, which was important in the regulation of flowering time in Arabidopsis; COLDAIR also trans-regulated the FLC gene by binding to protein complex PcG $[25,26]$. In addition, lncRNAs could be used as precursors of microRNAs (miRNAs), and some lncRNAs could also bind to miRNAs directly to regulate their functions [27]. Although a large number of lncRNAs has been identified in previous studies, the research on their biological functions is still in the initial stage, especially in plants.
Recently, high-throughput sequencing technology were often used to detect low-level expressed transcripts and identify numerous mRNAs, small RNAs and lncRNAs with important roles in biological processes $[15,16,28$, 29]. In our previous study, the expression patterns of genes and miRNAs in O. sativa, O. longistaminata and their three BIL progenies were performed, and the regulation of miRNAs on genes were also explored [2]. In this study, the high-throughput strand-specific RNA sequencing (ss-RNAseq) technology was used to study the expression differences and characteristics of lncRNAs and their target genes in O. sativa, O. longistaminata and their three BILs $\left(\mathrm{BC}_{2} \mathrm{~F}_{12}\right)$ progenies (L1710, L1817 and L1730). The lncRNAs acting as precursors or target mimics of miRNAs were also studied in these species. Further analysis showed that parental expression level dominance (ELD) phenomenon was the most common event in the three progenies. This work could provide valuable clues to reveal the molecular mechanisms of gene introgression of wild rice through hybrid and backcross.

\section{Results \\ Overview of the sequencing data}

To explore the expression characteristics of lncRNAs and their roles in O. sativa, O. longistaminata and their three BILs progenies at jointing-booting stage, the strandspecific RNA-seq (ssRNA-seq) technology was used in this study. Overall, an average of $13.70 \mathrm{~Gb}$ data with each sample was obtained and the gene expression correlations among three biological replicates were high, with average coefficient $\left(R^{2}\right)$ of 0.98 (Supplementary Fig. S1). On average, 101,808,785 (L1710), 91,859,276 (L1817), 88,566,772 (L1730), 98,879,278 (O. sativa) and 103,545,393 (O. longistaminata) raw reads were generated respectively, of which more than $94 \%$ reads were clean reads (Supplementary Table S1). All clean reads obtained from sequencing of 15 ssRNA libraries were uploaded to the NCBI's Sequence Read Archive (SRA) database (https://trace.ncbi.nlm.nih. gov/Traces/sra/sra.cgi?view=announcement) with accession numbers SRR9822767-SRR9822781. After reassembling and mapping, around $56 \%$ clean reads in $O$. longistaminata and $70 \%$ clean reads in three progenies and $O$. sativa were uniquely mapped to the rice reference genome (Supplementary Table S1) and 66,338 transcripts were identified as known mRNAs. Moreover, known mRNAs and transcripts whose information cannot be recognized were eliminated, and the remaining transcripts (described as novel transcripts in the following text) were further identified as candidates for lncRNAs. As a result, 16,038 novel transcripts were assembled, most of which were within $4500 \mathrm{nt}$ in length, and transcripts containing more than 10 exons accounted for a high proportion, while nearly half of the genes had only one transcript (Supplementary Table S2). 
Identification and the sequence characteristics of IncRNAs To identify lncRNAs in O. sativa, O. longistaminata and their three BIL progenies, the coding ability of the 16 , 038 novel transcripts were predicted using three software $(\mathrm{CPC}, \mathrm{CNCI}$ and txCdsPredict) and Pfam protein database. A total of 6719 novel lncRNAs were identified by predicting their coding ability (Fig. 1a), and then 1254 novel lncRNAs were screened out by the quantitative analysis with RSEM software (Fig. 1b, Table 1 and Supplementary Table S3). In addition, the sequence characteristics of identified lncRNAs were performed through comparing with that of mRNAs. The length of lncRNAs varied from 200 to $18,313 \mathrm{bp}$ with an average of $2348 \mathrm{bp}$, which was longer than that of known mRNAs (an average of $1708 \mathrm{bp}$ ) (Fig. 2a). About 50\% of lncRNAs were more than $2000 \mathrm{bp}$ in length, of which 42 lncRNAs were longer than $10,000 \mathrm{bp}$. The number of exons of the genes encoding lncRNAs was basically consistent with that of the known mRNA-coding genes, and $49.8 \%$ of lncRNA-coding genes and $53.2 \%$ of the known mRNAcoding genes contained 1-3 exons respectively (Fig. $2 \mathrm{~b}$ ). Most known mRNAs (87.1\%) and lncRNAs (70.1\%) were derived from genes having one or two transcripts (Fig. 2c). The GC content of lncRNA-coding genes varied from 23.45 to $78.93 \%$ with an average of $46 \%$, and most of them (79\%) with the GC content less than 50\% (Fig. 2d), while the GC content of known mRNA-coding genes varied from 28.73 to $84.16 \%$ with an average of 52.88 , and $55 \%$ of them with the GC content more than $50 \%$. Overall, the above results showed that the characteristics of lncRNA and mRNA sequences were diversified, for the length of lncRNAs was longer than that of known mRNAs, but the exon number of genes encoding lncRNAs was less than that of genes encoding mRNAs, and the GC content of lncRNA-encoding genes was also lower than that of known mRNA-encoding genes.

The expression level of lncRNAs and mRNAs were calculated using RSEM software. The number of lncRNAs and mRNAs expressed in O. sativa, O. longistaminata and their three BIL progenies was shown in Table 1, and the specific FPKM values for them were shown in Supplementary Table S3. As shown in Fig. 1b, $70.5 \%$ (884 of 1254) of lncRNAs expressed in all five lines. The number of lncRNAs which only expressed in one line was the most (35) in O. longistaminata and the least (6) in O. sativa (Fig. 1b). Furthermore, the distribution of expressed lncRNAs and mRNAs on 12 chromosomes was visualized using Circos (Fig. 2e \& f). The results showed that over 50\% lncRNAs and over $40 \%$ mRNAs were expressed on chromosomes 1 and 2, and the percentage of expressed lncRNAs was higher than that of expressed mRNAs on chromosomes 1, 2 and 11.

\section{Differentially expressed IncRNAs in five lines}

The FPKM values of lncRNAs and mRNAs (Supplementary Table S3) were used to analyze the differential expression of lncRNAs/mRNAs among $O$. sativa, O. longistaminata and their three BIL progenies with $\left|\log _{2} \mathrm{FC}\right| \geq 1$ and FDR $\leq 0.001$ by DEseq software. The analysis of the differentially expressed IncRNAs (DE-lncRNAs) was shown in Fig. 3. DElncRNAs were mostly up-regulated in progenies compared

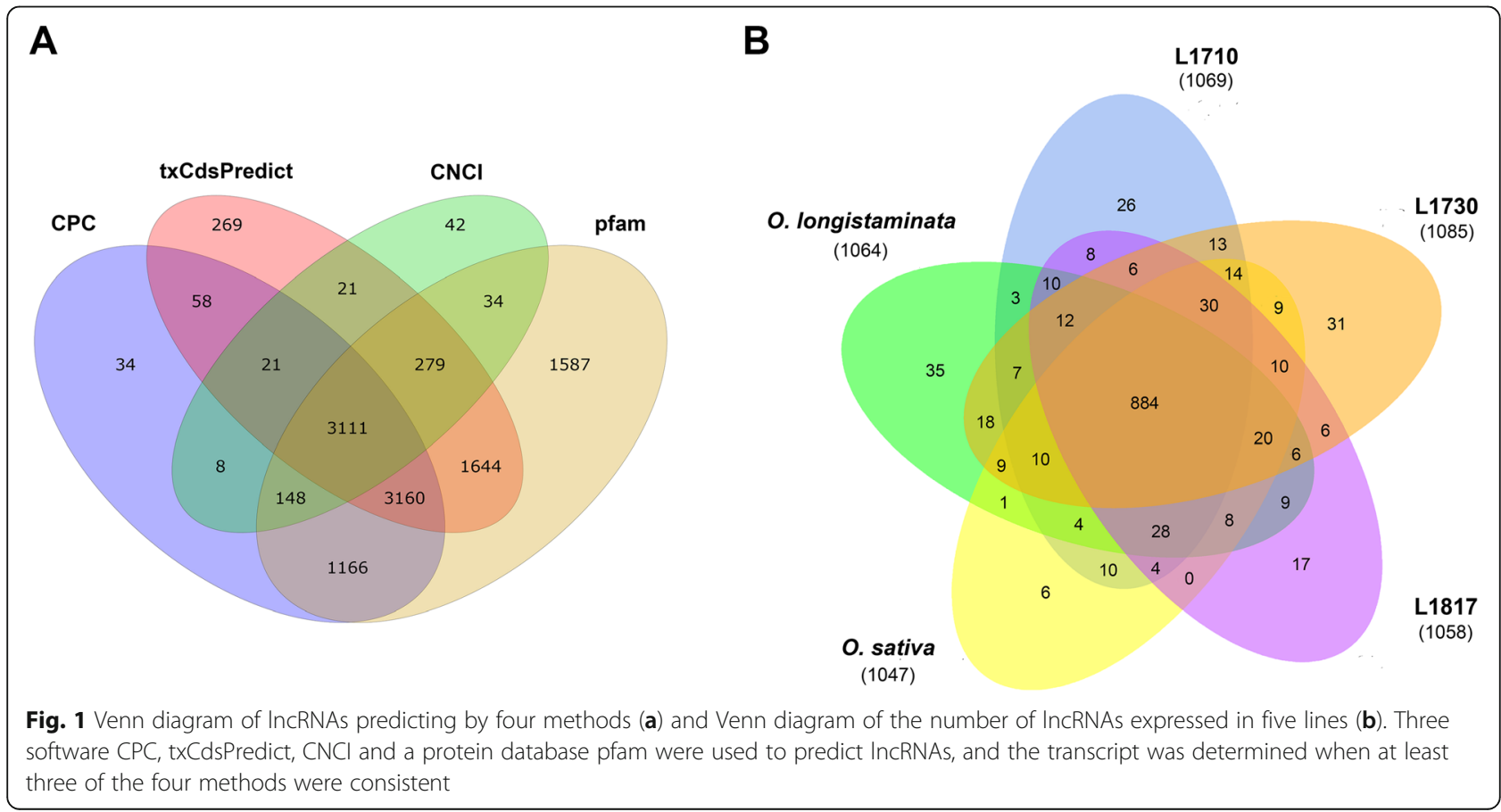


Table 1 The number of IncRNAs and mRNAs expressed 0. sativa, O. longistaminata and their three BIL progenies

\begin{tabular}{lll}
\hline Sample & Number of IncRNAs & Number of mRNAs \\
\hline L1710 & 1069 & 23,023 \\
L1817 & 1058 & 23,072 \\
L1730 & 1085 & 22,709 \\
O. sativa & 1047 & 22,448 \\
O. Iongistaminata & 1064 & 23,781 \\
\hline
\end{tabular}

with their parents (Fig. 3a). Furthermore, the DE-IncRNAs between progenies and O. longistaminata (an average of 458, and $69.2 \%$ of them were up-regulated in progenies) were significantly higher than that of progenies vs. O. sativa (an average of 267 , and $58.1 \%$ of them were up-regulated in progenies). Meanwhile, 41 and 185 common DE-lncRNAs were discovered in all three progenies compared with their two parents, respectively (Fig. $3 b$ \& d). Among these identified common DE-lncRNAs, the number of up-regulated lncRNAs were also higher than down-regulated lncRNAs in progenies (Fig. 3c \& e). The above results indicated that there was a greater difference between the three BIL progenies and the parent $O$. longistaminata, and the up-regulated DElncRNAs in BIL progenies might play critical roles. In the difference analysis among the three progenies, $299 \mathrm{DE}$ lncRNAs were found in L1710 vs. L1817, and 458 were found in L1710 vs. L1730 (Fig. 3a). This phenomenon was consistent with the fact that L1710 and L1730 have the largest difference in plant height. For a more detailed analysis, DE-lncRNAs with different fold changes $(\mathrm{FC}>2, \mathrm{FC}>10$,

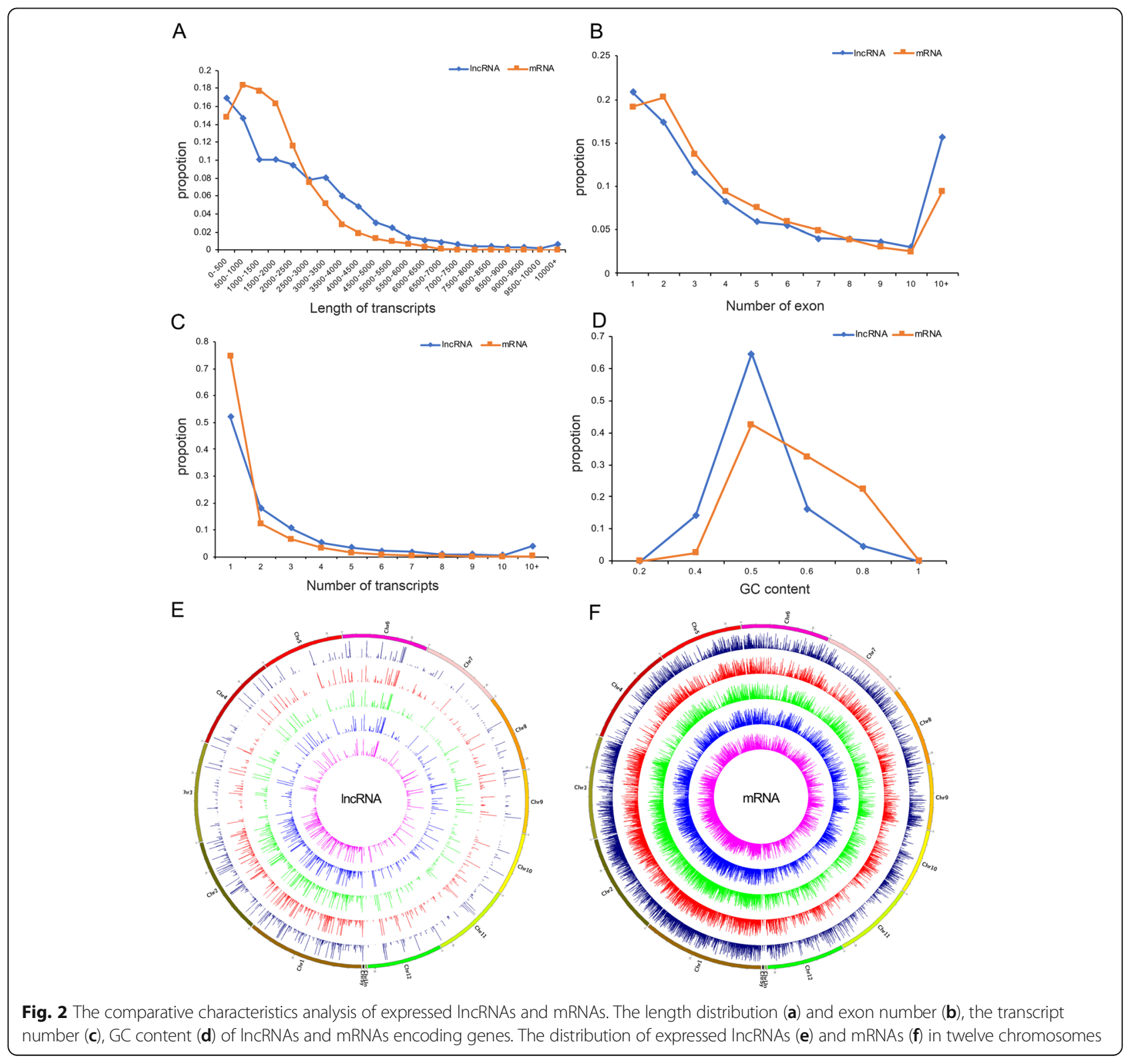



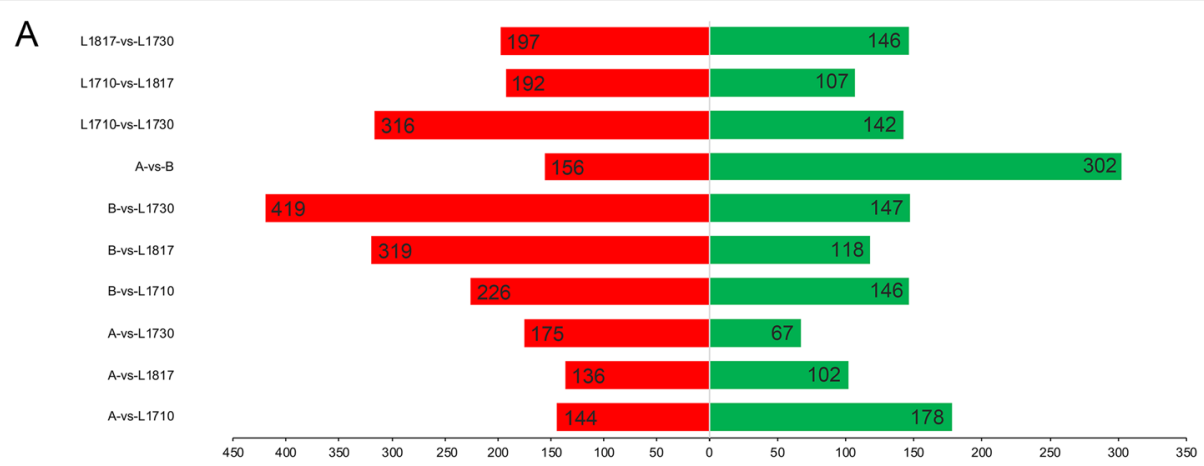

B
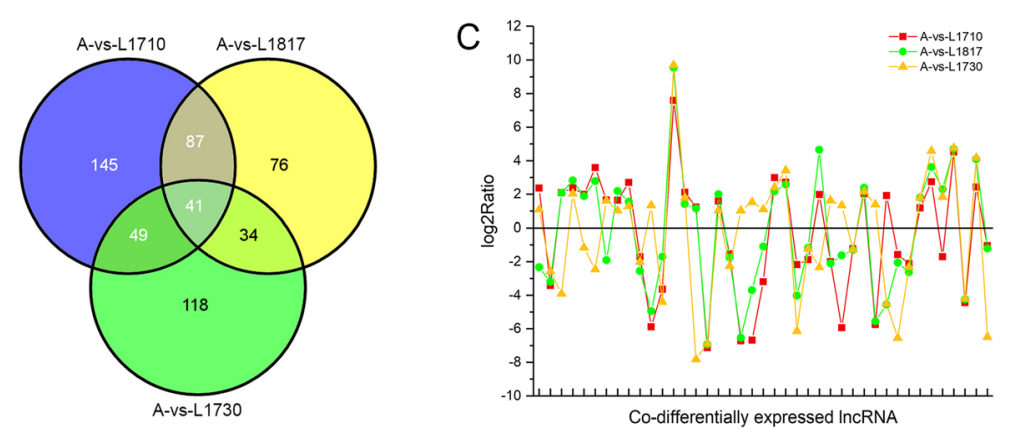

D
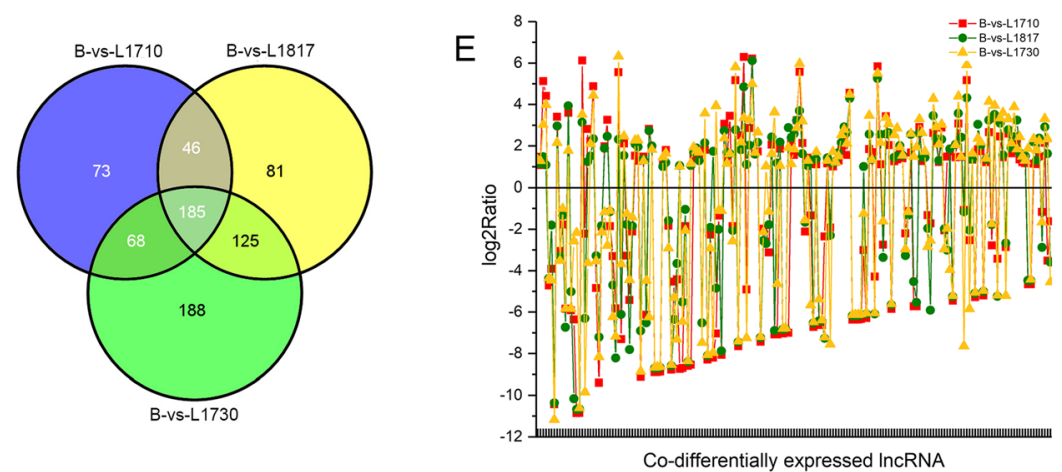

Fig. 3 Analysis of DE-IncRNAs. Number of DE-IncRNAs in all comparison groups (a). The red and green bars represented up- and down-expressed IncRNAs, respectively. Venn diagram of common DE-IncRNAs among three progenies and O. sativa (b) and the FC distribution of them (c). Venn diagram of common DE-IncRNAs among three progenies and O. longistaminata (d) and the FC distribution of them (e)

$\mathrm{FC}>50, \mathrm{FC}>100, \mathrm{FC}>200)$ in the three progenies compared with their parents were counted (Supplementary Fig. S2). With the increase of the FC of DE-lncRNAs, more lncRNAs were found in the comparison group with higher difference in plant height between progeny and parents. For example, among the comparison groups of three progenies and $O$. sativa, the number of DE-IncRNAs with $\mathrm{FC}>2$ was the most in L1710, while the number of DE-lncRNAs with FC > 50/100/200 was the largest in L1730.

\section{Prediction of target protein-coding genes of IncRNAs and their GO analysis}

One way in which lncRNAs perform their biological function is to regulate the expression of protein-coding genes through cis or trans interactions. LncRNAs may regulate the expression of target genes by one trans-regulated and three cis-regulated ways (cis_mRNA_up10k, cis_mRNA_ overlap and cis_mRNA_dw20k) (Fig. 4a). The Class cis_ mRNA_overlap could be further divided into 10 subclasses (Fig. 4b). Totally, the present data showed $89.5 \%$ (468 of 523) of lncRNAs regulated the expression of target genes through cis-acting, among which 45.3\% (212 of 468) belonged to the cis_mRNA_dw20k regulatory class. These results suggested that cis-regulation rather than transregulation was the main regulation type, and cis-regulation of IncRNAs located at $20 \mathrm{~kb}$ downstream of the target genes was the common cis-regulation type among the predicted lncRNA-mRNA regulation pairs in this study. As shown in Supplementary Table S4, a total of 431 lncRNAmRNA regulation pairs were detected in O. sativa, $O$. longistaminata and their three BIL progenies, among which 373 mRNAs were potential targets of 297 lncRNAs, 

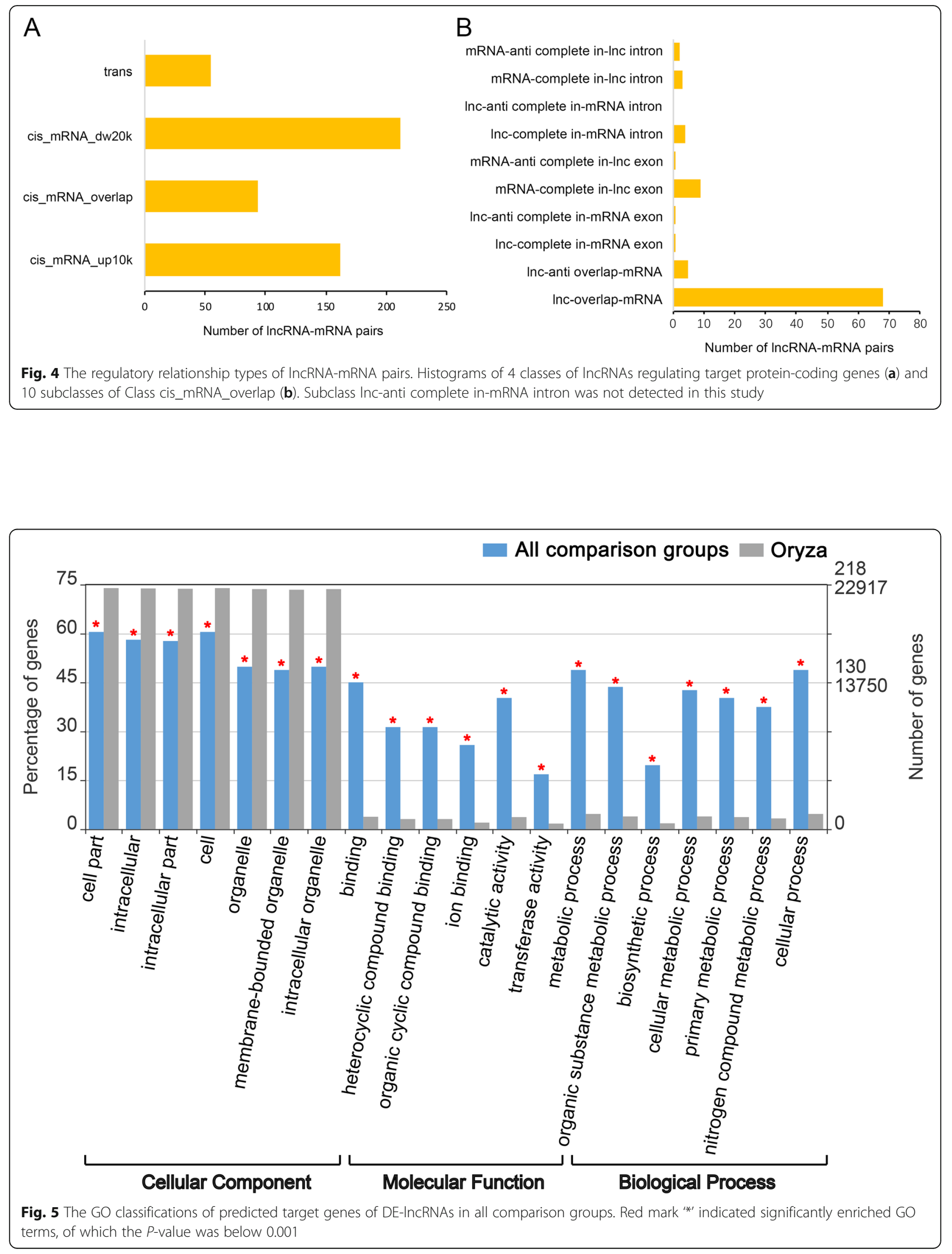
indicating that some lncRNAs may regulate multiple mRNAs at the same time.

In order to further understand the role of DElncRNAs among O. sativa, O. longistaminata and their three BIL progenies, GO enrichment analysis was conducted on the target genes of DE-lncRNAs in all comparison groups with rice genome as the reference (Fig. 5). Results showed that potential target genes of DE-lncRNAs were significantly enriched $(P<0.001)$ in 7 , 6 and 7 GO terms of cellular component, molecular function and biological process categories, respectively (Fig. 5). In addition, the percentage of potential target genes of DE-lncRNAs was lower than that of rice genome (the background) in significantly enriched GO terms of cellular component category, while higher in significantly enriched GO terms of molecular function and biological processes categories. This phenomenon indicated that DE-lncRNAs target genes that are significantly enriched in molecular function and biological processes categories might play important roles in regulating the growth and development of $O$. sativa, $O$. longistaminata and their three BIL progenies.

\section{Analysis of IncRNA acting as precursors of miRNAs}

LncRNAs could act as the precursors of microRNAs (miRNAs). To screen for miRNAs precursors in the five lines, the sequences of lncRNAs were aligned to the miRbase database using BLAST. As shown in Table 2, a total of 22 expressed lncRNAs were predicted to be the precursor of 20 miRNAs, of which 18 lncRNAs were DE-lncRNAs in five lines. Most lncRNAs acted as the precursor of one miRNA, while two of which (LTCONS_00035053, LTCONS_00007959) served as precursors of more than one miRNA (Table 2). Moreover, several lncRNAs might also be precursors for the same miRNA. For example, both LTCONS_00034708 and LTCONS_00034707 were the precursor of miR396c (Table 2). In addition, more than $50 \%$ of lncRNAs predicted as the precursors of miRNA were found to be transcribed from chromosome 2. RNAfold web server

Table 2 Prediction of IncRNA as miRNA precursor

\begin{tabular}{|c|c|c|c|c|}
\hline miRNA ID & IncRNA ID & Alignment length (nt) & Alignment ratio & Location \\
\hline miR162a & LTCONS_00029262 $^{\text {a }}$ & 171 & 1 & Chr2 \\
\hline miR166f & LTCONS_00014145 & 107 & 0.972 & Chr10 \\
\hline miR169a & LTCONS_00001063 & 173 & 1 & Chr1 \\
\hline miR394 & LTCONS_00029635 & 110 & 1 & $\mathrm{Chr} 2$ \\
\hline $\operatorname{miR396c}$ & LTCONS_00034708 & 141 & 1 & $\mathrm{Chr} 2$ \\
\hline miR396c & LTCONS_00034707 & 141 & 1 & $\mathrm{Chr} 2$ \\
\hline miR319b & LTCONS_00000824 & 197 & 1 & Chr1 \\
\hline miR166k & LTCONS_00035053 & 127 & 1 & Chr2 \\
\hline miR166h & LTCONS_00035053 & 119 & 1 & Chr2 \\
\hline miR393b & LTCONS_00051533 & 132 & 0.977 & Chr4 \\
\hline $\operatorname{miR} 172 d$ & LTCONS_00034806 & 130 & 1 & $\mathrm{Chr} 2$ \\
\hline $\operatorname{miR} 172 d$ & LTCONS_00034805 & 130 & 1 & $\mathrm{Chr} 2$ \\
\hline miR1430 & LTCONS_00024512 & 139 & 0.958 & Chr12 \\
\hline miR444b & LTCONS_00033178 & 138 & 1 & $\mathrm{Chr} 2$ \\
\hline miR444b & LTCONS_00033177 & 138 & 1 & Chr2 \\
\hline miR444d & LTCONS_00034231 & 143 & 1 & $\mathrm{Chr} 2$ \\
\hline miR1848 & LTCONS_00027106 & 69 & 0.921 & $\mathrm{Chr} 2$ \\
\hline miR1850 & LTCONS_00057871 & 133 & 0.985 & Chr5 \\
\hline miR1428b & LTCONS_00007959 & 124 & 1 & Chr1 \\
\hline miR1428d & LTCONS_00007959 & 124 & 0.919 & Chr1 \\
\hline miR1846e & LTCONS_00081175 & 69 & 0.957 & Chr9 \\
\hline miR1846e & LTCONS_00057746 & 69 & 0.928 & Chr5 \\
\hline miR396f & LTCONS_00034891 & 176 & 1 & $\mathrm{Chr} 2$ \\
\hline miR5083 & LTCONS_00009921 & 380 & 1 & Chr1 \\
\hline
\end{tabular}

${ }_{\text {indicated significantly differentially expressed IncRNAs }}$ 
was used to predict the secondary structures of lncRNAs and miRNA precursors to visualize the relationship of them. The predicted secondary structure of LTCONS 00057871 contained multiple stem-loop structures, and one of which was the potential precursor of miR1850 (Fig. 6). Mature miRNAs (miR1850.1, miR1850.2 and miR1850.3) were finally formed after the precursor was processed by enzymes.

\section{Analysis of IncRNA acting as decoys or target mimics of miRNAs}

LncRNAs could be used as decoys to directly or indirectly regulate the expression of target genes [30]. To further explore the roles of three kinds of RNAs (lncRNA, miRNA, and mRNA) in O. sativa, O. longistaminata and their three BIL progenies, interaction networks were constructed based on previous miRNA sequencing studies [2]. A total of 78 lncRNAs and 271 mRNAs were targeted by 280 miRNAs in the network (Supplementary Table S5). Statistic showed that $72.2 \%$ (202 of 280) miRNAs only targeted mRNAs (Supplementary Table S5). For example, osa-miR156k targeted 8 mRNAs (Fig. 7a), and three of them were found to be important in rice growth. Specifically, two mRNAs (LOC_Os02g04680.1 and LOC_ Os02g04680.2) were the two transcripts of OsSPL3 (SQLAMOSA PROMOTER-BINDING PROTEIN-LIKE3), which regulated root crown development in rice [31]. The other mRNA (LOC_Os08g39890.1) were the transcript of OsSPL14/IPA1, which could regulate DEP1 (DENSE AND ERECT PANICLE1), a critical gene influencing the plant height and panicle length [32]. Moreover, only a small fraction of miRNAs $(3.2 \%, 9$ of 280) only targeted lncRNAs (Supplementary Table S5, Fig. 7b). There were $24.7 \%$ (69 of 280) miRNAs targeted both mRNAs and lncRNAs (Supplementary Table S5). Further analysis found that only 5 miRNAs targeted more lncRNAs rather than mRNAs (Fig. 7c) and most miRNAs targeted more mRNAs rather than lncRNAs $(84.1 \%, 58$ of 69$)$ when miRNAs target both mRNAs and lncRNAs, which indicated that the binding ability of IncRNAs to miRNAs was weaker than that of mRNAs in most cases. For example, osa-miR172d-5p targeted 2 lncRNAs and 7 mRNAs (Fig. 7d), among which 3 mRNAs (LOC_Os03g51030.1, LOC_ Os03g51030.2 and LOC_Os03g51030.3) were the three

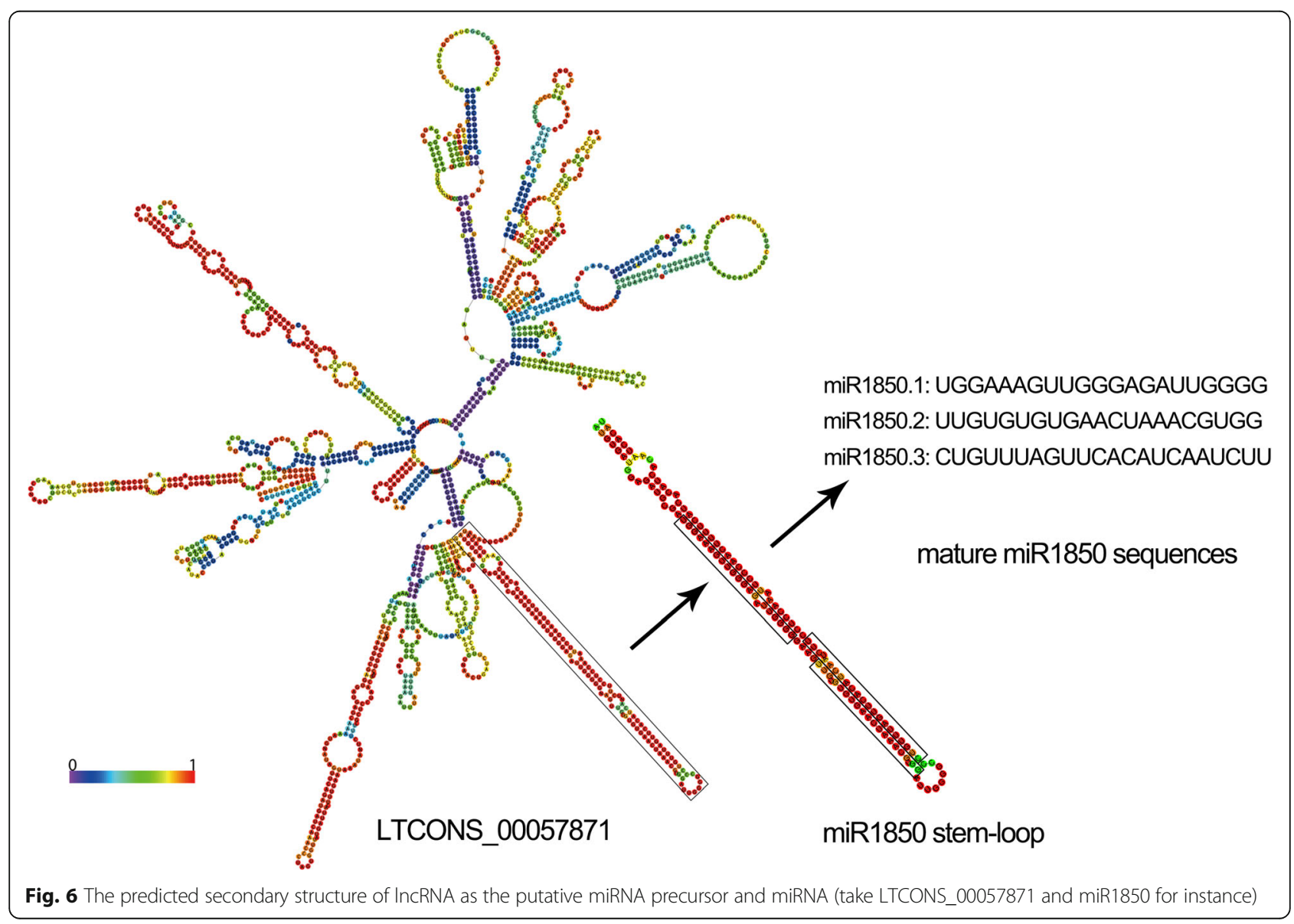



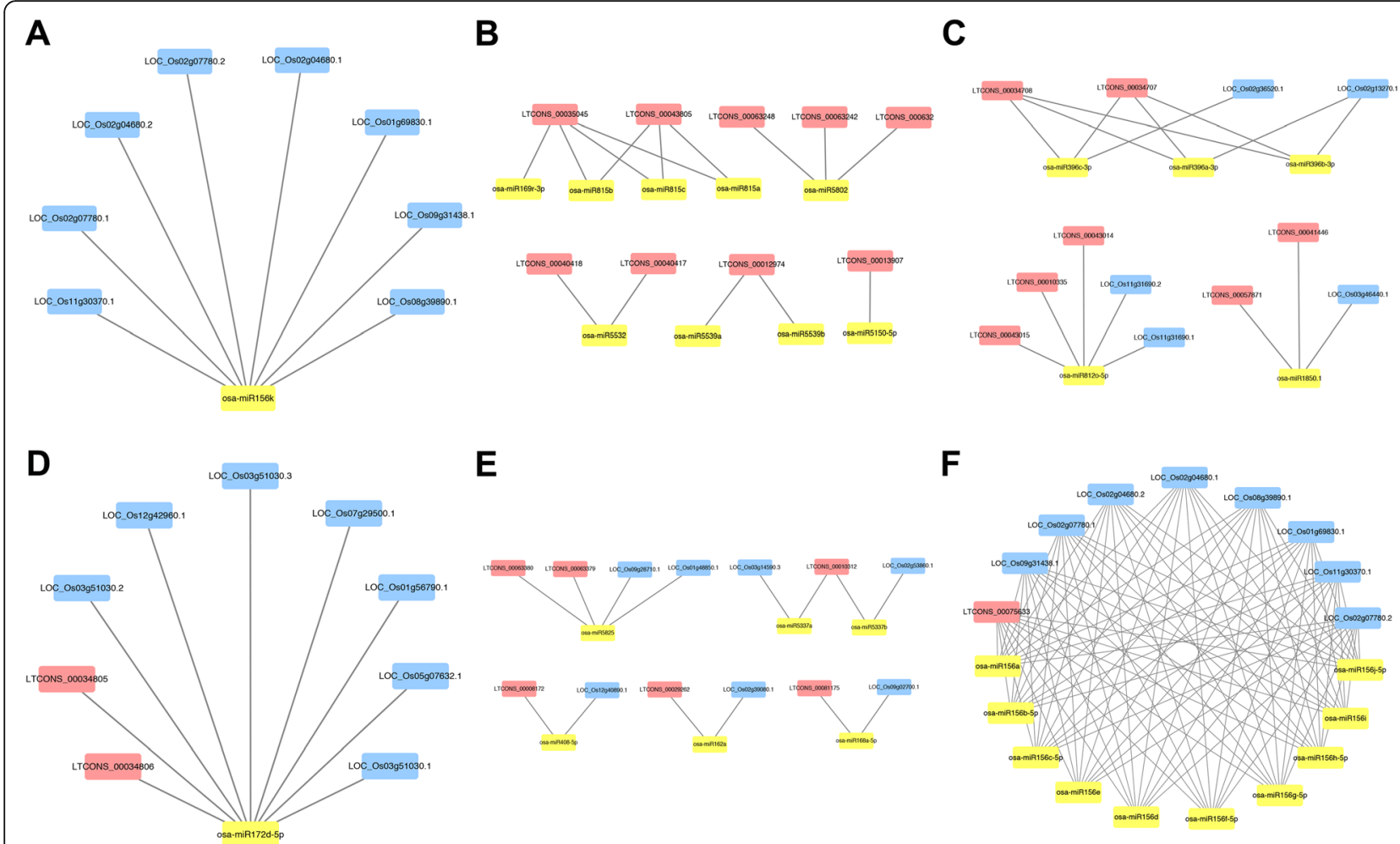

Fig. 7 The typical networks of IncRNAs, miRNAs and mRNAs

transcripts of PHYA (Phytochrome A), which played multiple roles in controlling the internode elongation in rice [33]. In addition, there were 6 miRNAs targeted with equal amounts of mRNAs and lncRNAs (Fig. 7e). Different miRNAs might also regulate multiple identical targets simultaneously (Fig. 7f). Interestingly, seven miRNAs were found to target their own potential precursor lncRNAs (Table 3), suggesting that these lncRNAs not only act as precursors of miRNAs, but also can bind with them to participate in the regulation of target gene expression.

\section{Parental expression level dominance analysis in three progeny lines}

Expression level dominance (ELD) refers to the expression level of some genes in the progeny close to that of one parent, but different from that of the other parent. Recently, many studies about hybrids and their parents have found that the expression of mRNAs showed parental ELD in progenies [2, 29, 34]. According to the criteria defined by Yoo et al. [34], gene expression patterns are divided into 12 categories, as shown in Fig. 8. Over $50 \%$ of lncRNAs showed parental ELD (category II, XI, IV and IX), and about $40 \%$ of lncRNAs showed transgressive up/down-regulation (category III, VII, X, V, VI and VIII) in all three progenies. Moreover, the number of lncRNAs showed parental ELD-A (A stands for $O$. sativa) was higher than that showed parental ELD-B (B stands for O. longistaminata) in all three progenies. To further get a glimpse of the possible biological functions of lncRNAs with ELD expression patterns, their potential target mRNAs were used for GO enrichment analysis. As shown in Supplementary Table S6, about 30\% of IncRNAs had potential target mRNAs in each progeny. The number of targets of IncRNAs showed ELD-A

Table 3 Analysis of miRNA targeting its precursor IncRNA

\begin{tabular}{|c|c|}
\hline miRNA ID & Targets \\
\hline miR162a & LTCONS_00029262 $^{a}$ \\
\hline miR393b & LTCONS_00051533 \\
\hline \multirow[t]{2}{*}{ miR396c } & LTCONS_00034708 \\
\hline & LTCONS_00034707 \\
\hline \multirow[t]{2}{*}{$\operatorname{miR} 172 d$} & LTCONS_00034806 \\
\hline & LTCONS_00034805 \\
\hline \multirow[t]{2}{*}{ miR1850 } & LTCONS_00057871 a \\
\hline & LTCONS_00041446 \\
\hline \multirow[t]{3}{*}{ miR444b } & LTCONS_00033178 \\
\hline & LTCONS_00033177 \\
\hline & LTCONS_00034231 \\
\hline \multirow[t]{3}{*}{ miR444d } & LTCONS_00034231 \\
\hline & LTCONS_00033177 \\
\hline & LTCONS_00033178 \\
\hline
\end{tabular}

andicated IncRNA acts as both precursor and target of corresponding miRNA 


\begin{tabular}{|c|c|c|c|c|c|c|c|c|c|c|c|c|}
\hline & \multicolumn{2}{|c|}{ Additivity } & \multicolumn{2}{|c|}{ ELD-A } & \multicolumn{2}{|c|}{ ELD-B } & \multicolumn{3}{|c|}{ Transgressive down-regulation } & \multicolumn{3}{|c|}{ Transgressive up-regulation } \\
\hline \multirow{2}{*}{ Category } & I & XII & II & XI & IV & IX & III & VII & $\mathrm{X}$ & $\mathrm{V}$ & VI & VIII \\
\hline & A-s-B & A-s-B & A-s-B & A-s-B & A-s-B & A-s-B & A-s-B & A-s-B & A-s-B & A-s-B & A-s-B & A-s-B \\
\hline \multirow[t]{2}{*}{$\mathrm{L} 1710$} & 23 & 16 & 97 & 82 & 28 & 89 & 39 & 30 & 15 & 50 & 26 & 59 \\
\hline & $4.2 \%$ & $2.9 \%$ & $17.5 \%$ & $14.8 \%$ & $5.1 \%$ & $16.1 \%$ & $7.0 \%$ & $5.4 \%$ & $2.7 \%$ & $9.0 \%$ & $4.7 \%$ & $10.6 \%$ \\
\hline \multirow[t]{2}{*}{ L1817 } & 26 & 12 & 157 & 81 & 32 & 56 & 12 & 10 & 15 & 74 & 35 & 54 \\
\hline & $4.6 \%$ & $2.1 \%$ & $27.8 \%$ & $14.4 \%$ & $5.7 \%$ & $9.9 \%$ & $2.1 \%$ & $1.8 \%$ & $2.7 \%$ & $13.1 \%$ & $6.2 \%$ & $9.6 \%$ \\
\hline \multirow[t]{2}{*}{ L1730 } & 2 & 16 & 202 & 92 & 17 & 25 & 17 & 18 & 13 & 113 & 27 & 87 \\
\hline & $0.3 \%$ & $2.5 \%$ & $32.1 \%$ & $14.6 \%$ & $2.7 \%$ & $4.0 \%$ & $2.7 \%$ & $2.9 \%$ & $2.1 \%$ & $18.0 \%$ & $4.3 \%$ & $13.8 \%$ \\
\hline
\end{tabular}

Fig. 8 Twelve expression patterns of IncRNAs in three progenies. A and B stand for O. sativa and O. longistaminata, respectively

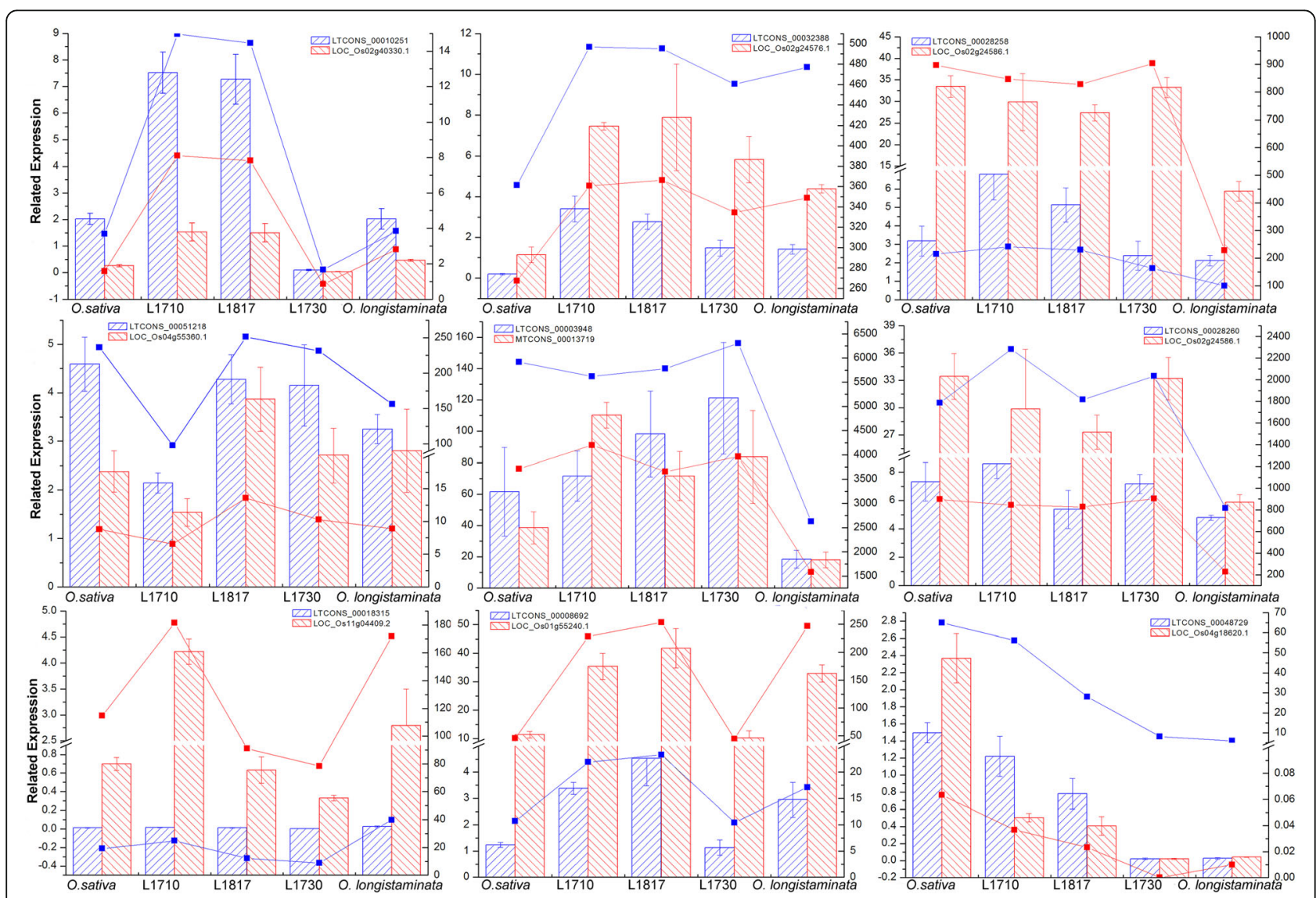

Fig. 9 Nine IncRNAs and their target genes were selected to verify the accuracy of the sequencing data using qRT-PCR in O. sativa, O. Iongistaminata and their three BIL progenies. The bar graph showed the results of qRT-PCR, and the broken line graph showed the results of sequencing, with IncRNAs in blue and mRNAs in red 
was higher than that showed ELD-B in all three GO categories (cellular component, molecular function and biological process) of L1817 and L1730 (Supplementary Fig. S3). However, the number of targets of lncRNAs showed ELD-A was less than that showed ELD-B in all three GO categories of L1710. In addition, ELD-A lncRNAs targets were enriched in 'cell', 'cell part' and 'organelle' term, while ELD-B lncRNAs targets were enriched in 'membrane' term in all three progenies (Supplementary Fig. S3). Furthermore, ELD-A lncRNAs targets were enriched in 'binding' term in L1710, while ELD-B IncRNAs targets were enriched in this term in L1817 and L1730 (Supplementary Fig. S3). ELD-A lncRNAs targets were enriched in 'cellular process' term in L1710 and L1730, while ELD-B lncRNAs targets were enriched in this term in L1817 (Supplementary Fig. S3). ELD-A lncRNAs targets were enriched in 'metabolic process' term in L1730, while ELD-B IncRNAs targets were enriched in this term in L1710 and L1817 (Supplementary Fig. S3). In conclusion, the non-addictive genes accounted for the majority in the comparison between BIL progenies and their parents, moreover, all three progenies (L1710, L1817 and L1730) biased towards O. sativa.

\section{Validation of the data by qRT-PCR}

To verify the accuracy of the sequencing data, 9 IncRNAs and their potential target genes were selected randomly for qRT-PCR analysis. Results showed that the sequencing data and the qRT-PCR results were basically consistent (Fig. 9), indicating that the sequencing data were reliable. The primers used for qRT-PCR were listed in Supplementary Table S7.

\section{Discussion}

In recent years, IncRNAs, a kind of regulatory RNAs, have become a research hotspot. With the development of sequencing technology, lncRNAs have been found to play critical roles in plant growth and sexual reproduction [14, 35]. Specifically, studies have shown that lncRNAs were involved in female/male sterility of plants [36-39], and plant stress response process $[40,41]$. So far, there have been no reports on the IncRNA expression patterns and their functions in BIL progenies relative to their parents. Based on our previous studies on the mRNA and miRNA expression patterns in $O$. sativa, O. longistaminata and their three BIL progenies [2], the study on lncRNA expression patterns will help us to further understand the regulatory factors for differential expression of genes in BIL progenies relative to their parents.

LncRNAs might be involved in regulating the expression of plant hormone-related genes in 0 . sativa, $O$. longistaminata and their BIL progenies

Plant hormones can regulate the physiological responses of plant growth, development and differentiation independently or in a variety of coordinated ways. Plant hormone content are affected by related gene expressions, while the later are regulated at transcriptional and post-transcriptional levels by various factors [29, 42, 43]. Many lncRNAs with down-regulated polyadenylation (DPA) participated in the biosynthesis, transport and metabolism of $\mathrm{ABA}$ in rice, thereby activating the expression of a series of stress response genes [44]. As described below, some lncRNAs targeted gibberellin (GA), ethylene and auxin related genes in this study, and their regulatory effects might have an impact on growth and development of rice. LTCONS_00063919 targeted D35/ OsKO2 (LOC_Os06g37364, Supplementary Table S4), which encodes an ent-kaurene oxidase (KO) of catalytic gibberellin biosynthesis, and rice without this gene shows a severe dwarf-phenotype [45]. SLRL1 (LOC_ Os01g45860), a member of GRAS gene family, was targeted by LTCONS_00002962 (Supplementary Table S4), and GA induces the expression of SLRL1, the overexpression of which will also lead to the dwarf-phenotype in rice [46]. GID2 (LOC_Os02g36974) was a target gene of LTCONS_00033182 (Supplementary Table S4), which regulate the degradation of an inhibitory factor (SLR1) in GA signal transduction, and rice presented a severe dwarfing phenotype when GID2 gene was mutated [47]. Furthermore, LTCONS_00032876 was predicted to target OsCTR2 (LOC_Os02g32610, Supplementary Table S4), and the Raf-like protein CONSTITUTIVE TRIPLE RESPONSE1 (CTR1) was involved in ethylene receptor signal transduction to regulate multiple growth and development processes in rice [48]. In addition, OsARF24 was a target gene of LTCONS_00025454 (Supplementary Table S4), and the low expression of OsARF23 and OsARF 24 would reduce the response of rice to auxin, thereby affecting the growth and morphogenesis of rice [49]. Therefore, lncRNAs that target to plant hormonerelated genes might regulate plant growth and development and affect the plant height in O. sativa, O. longistaminata and their BIL progenies.

\section{LncRNAs might regulate the adaptability of $O$. sativa, $O$. longistaminata and their BIL progenies}

Studies have shown that lncRNAs are involved in plant responses to various biotic and abiotic stresses [40, 44, 50]. Jain et al. [41] identified many lncRNA candidate genes from 24 blast resistant rice lines, revealing their regulatory roles in rice blast resistance. Many lncRNAs with DPA may play important roles in the growth of rice under various abiotic stresses (such as heat, cold, drought and salt stress) [44]. As shown in Supplementary Table S4, LTCONS_00033755 targeted trehalose-6phosphate phosphatase (OsTPP1, LOC_Os02g44230), which was a member of TPS/TPP gene family. The overexpression of OsTPP1 could enhance the tolerance to 
cold and salt stress, and simultaneously activated the expression of multiple stress response genes in rice [51]. The protein encoded by GF14e (LOC_Os02g36974, a targeted gene of LTCONS_00033182, Supplementary Table S4) affects the expression of defense response genes, cell death and resistance to bacterial blight and sheath blight in rice [52]. Meanwhile, the protein encoded by OsGF14e, which was regulated by WRKY71, positively regulated rice resistance to panicle blast [53]. In addition, WRKY13 (LOC_Os01g54600) was targeted by LTCONS_00010291 (Supplementary Table S4) in this study, and it is well known that WRKY13 could directly inhibited WRKY42, which could negatively regulate the response of rice to blast fungus (Magnaporthe oryzae) by inhibiting JA signaling related genes [54]. Moreover, OsPCF5 was targeted by LTCONS_00010204 (Supplementary Table S4), and as a member of TCP transcription factor family, OsPCF5 plays a negative role in response to low temperature stress in rice [55]. Thus, four lncRNAs mentioned above might regulate the expression of disease-resistance related genes in O. sativa, $O$. longistaminata and their BIL progenies, and thereby affecting their adaptability.

LncRNAs might regulate the growth of $O$. sativa, $O$. longistaminata and their BIL progenies by competitively binding miRNAs to mRNAs

Some lncRNAs bind to miRNAs as decoys, so that these miRNAs cannot bind to their target genes normally, thereby affecting their regulation of target genes [20]. Two lncRNAs were found to adsorb miR160 and miR164 respectively in anther, pistil and seed of rice [35]. In this study, LTCONS 00001063 was predicted to competitively bind miR169f.1 and miR169o with OsHAP2G (LOC_Os07g41720) and OsHAP2H (LOC_Os03g44540), which were the two coding genes of the HAP2 subunit of HAP complex [56]. Thus, it is speculated that LTCONS_00001063 might participate in the regulation of the HAP2 subunit gene expression during the growth and development of rice. Moreover, LTCONS 00034806 and LTCONS_00034805 were predicted to competitively bind miR172d-5p with Phytochrome A (PHYA; LOC_Os03g51030), which can regulate the elongation of rice nodes and play critical role in the vegetative growth stage of rice [33, 57]. Therefore, LTCONS_00001063, LTCONS 00034806 and LTCONS_00034805 may regulate the expression level of miRNA target genes by competitively binding miRNAs with specific mRNAs, and then participate in the growth process of stem at the jointing stage of rice.

\section{The difference of IncRNA expression between BIL} progenies and $O$. sativa is smaller than that between BIL progenies and $O$. longistaminata

Our previous study mainly explored the gene expression and miRNA regulation in $O$. sativa, $O$. longistaminata and their three BIL progenies [2]. In this study, expression characteristics of lncRNAs and their potential target genes were investigated in these species. These two studies found something in common. For example, the previous study showed that the number of DEGs between progenies and $O$. longistaminata was higher than that between progenies and $O$. sativa. Similarly, the number of DE-lncRNAs between progenies and O. longistaminata was also higher than that between progenies and $O$. sativa in this study. In addition, the previous study showed that most genes displayed ELD of one parent and more ELD-A (A stands for O. sativa) genes than ELD-B (B stands for O. longistaminata) genes were observed in three progenies. Analogously, over $50 \%$ of lncRNAs showed parental ELD and the number of lncRNAs showed parental ELD-A was higher than that showed parental ELD-B in all three progenies in this study. The above results indicated that the difference of lncRNA expression between BIL progenies and O. sativa is smaller than that between BIL progenies and O. longistaminata. As indicated in previous studies, most of the chromosome complements of the BIL progenies were inherited from the recurrent parent $O$. sativa [2]. Therefore, the most likely reason for the difference of lncRNA expression was the genetic background difference between BIL progenies and each of their parent.

\section{Conclusions}

In this study, high-throughput ss-RNA-seq technology was used to explore the functional characteristics and difference of lncRNAs in $O$. sativa, $O$. longistaminata and their three BIL progenies. LncRNAs were identified and most of them expressed in all five lines. The analysis of DE-lncRNAs showed that the difference of lncRNAs between the three progenies and $O$. sativa, including the number and the fold change of DE-lncRNAs, was greater than that between the three progenies and O. longistaminata. In addition, most DE-lncRNAs were up-regulated in progenies compared with their parents. Some lncRNAs could regulated the expression of mRNAs, most of which was cis-regulation. Most miRNAs targeted more mRNAs rather than lncRNAs when both mRNAs and lncRNAs were targeted. Some miRNAs were found to target their own potential precursor lncRNAs. Furthermore, more than half of the lncRNAs showed parental ELD in all three progenies, and the number of lncRNAs showed parental ELD-O. sativa was higher than that showed parental ELD-O. longistaminata, which indicated that all three progenies were biased towards $O$. sativa. Further analysis showed that lncRNAs might be involved in regulating the expression of plant hormone-related genes in all five lines, and also can regulate the adaptability of them. Taken together, these results provided valuable clues for elucidating the 
functional features and expression differences of lncRNAs between $O$. sativa, $O$. longistaminata and their BIL progenies, and expanded our understanding of the biological functions of lncRNAs in rice.

\section{Methods}

\section{Plant materials}

Oryza sativa ssp. indica cv. 9311, O. longistaminata and their three BILs $\left(\mathrm{BC}_{2} \mathrm{~F}_{12}\right)$ progenies (L1710, L1817 and L1730) were used as plant materials in this study. These materials were all obtained from Dr. Shaoqing Li's laboratory in College of Life Sciences, Wuhan University, Wuhan, China. The construction of $\mathrm{BC}_{2} \mathrm{~F}_{12}$ consists of 4 steps: 1) O. sativa (maternal) was crossbred with $O$. longistaminata (paternal) to generate F1 hybrid; 2) F1 hybrid (paternal) was backcrossed with O. sativa (maternal) to generate $\mathrm{BC}_{1} \mathrm{~F}_{1}$; 3) $\mathrm{BC}_{1} \mathrm{~F}_{1}$ individuals (paternal) were backcrossed with $O$. sativa (maternal) to generate $\mathrm{BC}_{2} \mathrm{~F}_{1}$; 4) $\mathrm{BC}_{2} \mathrm{~F}_{1}$ finally produces $\mathrm{BC}_{2} \mathrm{~F}_{12}$ through 11 generations of self-fertilization by the single seed descent method. The genome composition of three progenies was basically the same, and most of which were inherited from the cultivated rice (O. sativa), while only about 10 to $15 \%$ of which was inherited from O. longistaminata [2]. As Cao et al. [2] described, three BIL progenies had different plant heights. Specifically, the plant height of 5 lines at mature stage was ranked as $\mathrm{L} 1710<O$. sativa $<$ $\mathrm{L} 1817<$ O. longistaminata $<$ L1730 [2]. Germinated seeds of progenies and $O$. sativa were sown in soil and then the seedlings were transplanted into plots after 30 days in the greenhouse of Wuhan University, Wuhan, China. The rhizomes of $O$. longistaminata were also planted in plots. Stems from five lines with three biological replicates at jointing-booting stage were harvested and immediately stored in liquid nitrogen for subsequent RNA extraction.

\section{Construction of RNA libraries and sequencing}

The total RNAs of stems in five lines were extracted using TRIzol reagent according to the manufacturer's protocal (Invitrogen, Carlsbad, CA, USA). The purity, concentration and $\mathrm{OD}_{260} / \mathrm{OD}_{280}$ ratio of total RNAs in each sample were detected by Agilent 2100 Bioanalyzer (Agilent RNA 6000 Nano Kit). The ribosomal RNAs (rRNAs) were removed from the total RNAs using the Ribo-Zero $^{\text {Tw }}$ rRNA removal kit, and then the RNAs were randomly fragmented. The first-strand cDNA was synthesized by reverse transcription with the fragmented RNA as the template and a random six-base sequence as the primer, and the second-strand cDNA was subsequently synthesized by replacing dTTP with dUTP. The sequencing libraries were then constructed through the cDNA end repair, adding poly A-tailing and adapter, Uracil-N-Glycosylase digestion, and several rounds of
PCR amplification. Quality control and quantification analysis were performed on all libraries. Finally, the fifteen libraries were sequenced using the Illumina HiSeq 4000 platform and 150 bp paired-end reads were generated. Each line was sequenced with three biological replicates.

\section{Data filtering, sequence alignment and assembly}

To further ensure that rRNA was not presented in raw reads, reads were aligned to the ribosomal database using the short reads alignment tool SOAPnuke (v1.5.2) [58] and the aligned reads were removed (up to 5 mismatches were allowed). After removing reads with adaptor, $\mathrm{N}$ ratio containing greater than $10 \%$ and inferior quality, the filtered reads were aligned to the reference genome of rice (https://www.ncbi.nlm.nih.gov/ assembly/GCF_001433935.1) using HISAT2 software (v2.0.4) [59] and re-assembled using StringTie (v1.0.4) [60]. To obtain the positional relationship of reassembled transcripts, they were compared with known mRNAs and lncRNAs using cuffcompare, which was one of the tools of Cufflinks (v2.2.1) [61], and then the final transcripts were combined using Cuffmerge (one tool of Cufflinks, v2.2.1) [61].

\section{Identification of mRNAs and IncRNAs}

LncRNAs was identified with reference to the study of Liu et al. [39]. The coding ability of combined transcripts (FPKM $\geq 0.5$, Coverage $>1$, Length $>200$ ) were predicted using protein database Pfam (http://pfam.xfam.org/) [62] and three software, including Coding Potential Calculator (CPC, v0.9r2, http:// CPC.cbi.pku.edu.cn) [63], Coding-Non-Coding Index (CNCI, https://github.com/www-bioinfo-org/CNCI) [64] and txCdsPredict (http://hgdownload.soe.ucsc.edu/ admin/jksrc.zip). The four judgment methods are elaborated as follows: 1) If the transcripts were mapped to the Pfam database, they were recognized as mRNAs, otherwise lncRNAs; 2) CPC_threshold $=0$, transcripts which have values greater than 0 were mRNAs, otherwise lncRNAs; 3 ) CNCI_threshold $=0$, transcripts which have values greater than 0 were mRNAs, otherwise lncRNAs; 4) txCdsPredict threshold $=500$, transcripts which have values greater than 500 were mRNAs, otherwise lncRNAs. Transcripts were finally identified as mRNAs or IncRNAs when at least three of the four above methods were consistent.

\section{Differential expression analysis of IncRNAs}

Clean reads were aligned to the reference genome using Bowtie2 software (v2.2.5) [65] and then the expression levels of transcripts were calculated using RSEM (v1.2.12) [66]. The normalized method used by RSEM software was FPKM, and the formula was as follows: $\mathrm{FPKM}=\frac{10^{6} \mathrm{C}}{N L / 10^{3}}$. In this formula, ' $\mathrm{C}$ ' is the number of 
unique fragments for the target gene, ' $\mathrm{N}$ ' is the total number of fragments which were uniquely matched the reference genome, and ' $\mathrm{L}$ ' is the total number of bases in the coding region of the target gene. The calculated FPKM values, representing the gene expression levels, can be directly used to compare the gene expression differences among different samples. Correlations for three biological replications were calculated based on FPKM values using cor function in $\mathrm{R}$ (v3.3.0, https://www.rproject.org/). Software DEGseq [67] was used to analyze the difference of the comparison group. In this study, transcripts that exhibited fold change $(F C) \geq 2$ $\left(\left|\log _{2} \mathrm{FC}\right| \geq 1\right)$ and the false discovery rate $(\mathrm{FDR}) \leq 0.001$ were regarded as significantly differentially expressed transcripts. The differentially expressed IncRNAs were screened from the differentially expressed transcripts according to the ID of lncRNAs.

\section{Identification of IncRNAs target genes and GO analysis} LncRNAs regulate target genes in two ways, including cis- and trans-regulation. When lncRNA plays the cisregulatory role, the location of lncRNA on the chromosome is close to the target gene, so the mRNA adjacent to lncRNA is selected as its target gene; when lncRNA plays the trans-regulatory role, it does not depend on the position relationship with the target gene, and its target gene can be predicted by calculating the binding energy. Specifically, according to the study of Liu et al. [39], target genes were analyzed in three steps: 1) the correlations between all identified lncRNAs and mRNAs were analyzed statistically (spearman values $\geq 0.6$ and Pearson values $\geq 0.6$ ); 2) IncRNAs were determined to play cis-regulatory roles when they located within $10 \mathrm{k}$ upstream or $20 \mathrm{k}$ downstream of the target genes; 3 ) when lncRNAs were not located in that range, RNAplex was used to analyze the binding energies of lncRNAs and mRNAs. If the binding energies were less than -30 , they were determined as lncRNAs with trans-regulatory effects. Furthermore, if IncRNA overlaps with the target gene, it will be further classified to 10 subclasses (such as lnc-overlap-mRNA and lnc-anti overlap-mRNA), which is conducive to increasing our understanding of the cis-regulated details of IncRNA $[68,69]$. In addition, all identified potential target genes of differentially expressed lncRNAs (DE-lncRNAs) in all comparison groups were used for $\mathrm{GO}$ enrichment analysis using the WEGO website (http://wego.genomics.org.cn) with rice genome as the background.

\section{Prediction of IncRNA as the miRNA precursor}

To predict lncRNAs that might be the precursors of the microRNAs (miRNAs), BLAST tool was used to align all lncRNAs to the miRbase (http://www.mirbase.org) [70]. The lncRNA was recognized as miRNA precursor when the coverage ratio of IncRNA sequence to the miRNA precursor sequence was over $90 \%$, and the precursor sequences of miRNAs were from the previous data [2]. The secondary structures of lncRNA and miRNA precursor were plotted by RNAfold web server (http://rna. tbi.univie.ac.at/cgi-bin/RNAWebSuite/RNAfold.cgi). The interactive network relationships of lncRNA, miRNA and mRNA were displayed by Cytoscape software (v3.7.1, http://www.cytoscape.org).

\section{Validation of the data by qRT-PCR}

To verify the accuracy of lncRNA sequencing data in this study, 9 lncRNAs and their predicted potential target genes were randomly selected from all expressed lncRNAs and verified by quantitative Real-Time PCR (qRT-PCR). Total RNAs of stems from five lines were extracted using TRIzol reagent (Invitrogen), and reverse transcribed with random primers. The lncRNA/mRNA specific primers were designed using Primer 5.0 software (http://www.premierbiosoft.com/index.html). The PCR amplifications were conducted using the SYBR@qPCR Mix (Toyobo) in the ABI Step One Plus Real-Time PCR System (Applied Biosystems, USA). The qRT-PCR reaction was processed as previously described with three biological replicates and three technical repeats [29]. In addition, OsActin1 [71] was employed as internal control gene to normalize each lncRNA/mRNA threshold cycle reaction.

\section{Supplementary information}

Supplementary information accompanies this paper at https://doi.org/10. 1186/s12870-020-02508-y.

\section{Additional files 1: Figure S1. Correlation coefficients for three} biological replicates of gene expression data

Additional files 2: Figure S2. Differentially expressed IncRNAs in three progenies compared with their parents. FC stands for the fold change of DE-IncRNAs. The red number represents the number of up-regulated DEIncRNAs and the blue number represents the number of down-regulated DE-IncRNAs

Additional files 3: Figure S3. The GO enrichment analysis of potential target mRNAs of IncRNAs with ELD expression patterns in L1710 (A),

L1817 (B) and L1730 (C). Red mark ${ }^{* \prime \prime}$ indicated significantly enriched GO terms, of which the $P$-value was below 0.05

Additional files 4: Table S1. Summary of sequencing reads in fifteen libraries

Additional files 5: Table S2. The statistical analysis of assembled 16,038 novel transcripts

Additional files 6: Table S3. The normalized FPKM values for all IncRNAs and mRNAs in all samples

Additional files 7: Table S4. The regulatory relationship types of IncRNA-mRNA pairs

Additional files 8: Table S5. Targeting relationships between miRNAs and IncRNAs/mRNAs

Additional files 9: Table S6. Parental expression level dominance IncRNAs and its target mRNAs in three progenies

Additional files 10: Table S7. The primers used for quantitative realtime PCR 


\section{Abbreviations}

ABA: Abscisic acid; BILs: Backcross introgression lines; CNCl: Coding-NonCoding Index; CPC: Coding Potential Calculator; CTR1: CONSTITUTIVE TRIPLE RESPONSE1; DE-IncRNAs: Differentially expressed IncRNAs; DEP1: DENSE AND ERECT PANICLE1; DPA: Polyadenylation; ELD: Expression level dominance; FC: Fold change; FDR: False discovery rate; FLC: FLOWERING LOCUS C; GA: Gibberellin; KO: Ent-kaurene oxidase; IncRNA: Long non-coding RNA; miRNAs: micrornas; OSTPP1: Trehalose-6-phosphate phosphatase; Phytochrome A: PHYA; qRT-PCR: Quantitative Real-Time PCR; rRNAs: Ribosomal RNAs; OSSPL3: SQUAMOSA PROMOTER-BINDING PROTEIN-LIKE3; SRA: Sequence Read Archive; ss-RNAseq: Strand-specific RNA sequencing.

\section{Acknowledgments}

Not applicable.

\section{Authors' contributions}

JW and AC conceived and designed the study. ML and AC performed most of bioinformatics analysis and analyzed most of the data. ZL performed some bioinformatics analysis. RW performed the gRT-PCR experiment. SL created the $\mathrm{BC}_{2} \mathrm{~F}_{12}$ lines and provided the experimental materials, proposed some critical suggestions for data analysis and revised the manuscript. AC were responsible for planting materials. ML wrote the manuscript with contributions from all the authors. JW revised the manuscript. All authors read and approved the final manuscript.

\section{Funding}

This work was supported by the State Key Basic Research and Development Plan of China (2013CB126900). The funding body played no role in the experimental design, collection, analysis, and interpretation of data and preparation of the manuscript

\section{Availability of data and materials}

The datasets analyzed during the current study are available in the NCBI's Sequence Read Archive (SRA) repository (https://trace.ncbi.nlm.nih.gov/ Traces/sra/sra.cgi?view=announcement) and the accession numbers of the fifteen runs are SRR9822767-SRR9822781. The web link of the reference genome of rice was https://www.ncbi.nlm.nih.gov/assembly/GCF_001433935.1. The web link of protein database Pfam was http://pfam.xfam.org/. The web link of miRbase was http://www.mirbase.org. All other data generated or analyzed during this study were included in this published article and the additional files.

\section{Ethics approval and consent to participate} Not applicable.

\section{Consent for publication}

Not applicable.

\section{Competing interests}

The authors declare that they have no competing interests.

\section{Received: 27 November 2019 Accepted: 22 June 2020}

\section{Published online: 29 June 2020}

\section{References}

1. Ge S, Sang T, Lu BR, Hong DY. Phylogeny of rice genomes with emphasis on origins of allotetraploid species. Proc Natl Acad Sci U S A. 1999:96: 14400-5.

2. Cao A, Jin J, Li S, Wang J. Integrated analysis of mRNA and miRNA expression profiling in rice backcrossed progenies $\left(\mathrm{BC}_{2} \mathrm{~F}_{12}\right)$ with different plant height. PLoS One. 2017;12:e0184106

3. Ma X, Fu Y, Zhao X, Jiang L, Zhu Z, Gu P, Xu W, Su Z, Sun C, Tan L. Genomic structure analysis of a set of Oryza nivara introgression lines and identification of yield-associated QTLs using whole-genome resequencing. Sci Rep. 2016;6:27425.

4. Li X, Guo W, Wang B, Li X, Chen H, Wei L, Wang Y, Wu J, Long H. Instability of chromosome number and DNA methylation variation induced by hybridization and amphidiploid formation between Raphanus sativus $L$ and Brassica alboglabra Bailey. BMC Plant Biol. 2010;10:207.
5. Chen Z, Hu F, Xu P, Li J, Deng X, Zhou J, Li F, Chen S, Tao D. QTL analysis for hybrid sterility and plant height in interspecific populations derived from a wild rice relative, Oryza longistaminata. Breed Sci. 2009;59:441-5.

6. Yugander A, Sundaram RM, Singh K, Ladhalakshmi D, Subba Rao LV, Madhav MS, Badri J, Prasad MS, Laha GS. Incorporation of the novel bacterial blight resistance gene Xa38 into the genetic background of elite rice variety improved Samba Mahsuri. PLoS One. 2018;13:e0198260.

7. Saikumar S, Gouda PK, Saiharini A, Varma CMK, Vineesha O, Padmavathi G, Shenoy W. Major QTL for enhancing rice grain yield under lowland reproductive drought stress identified using an O. sativa/O. glaberrima introgression line. Field Crops Res. 2014;163:119-31.

8. Moumeni A, Satoh K, Venuprasad R, Serraj R, Kumar A, Leung H, Kikuchi S. Transcriptional profiling of the leaves of near-isogenic rice lines with contrasting drought tolerance at the reproductive stage in response to water deficit. BMC Genomics. 2015:16:1110.

9. Brog YM, Osorio S, Yichie Y, Alseekh S, Bensal E, Kochevenko A, Zamir D, Fernie AR. A Solanum neorickii introgression population providing a powerful complement to the extensively characterized Solanum pennellii population. Plant J. 2019;97:391-403.

10. Song WY, Wang GL, Chen LL, Kim HS, Pi LY, Holsten T, Gardner J, Wang B, Zhai WX, Zhu LH, Fauquet C, Ronald P. A receptor kinase-like protein encoded by the rice disease resistance gene, Xa21. Science. 1995;270:1804-6.

11. Hu FY, Tao DY, Sacks E, Fu BY, Xu P, Li J, Yang Y, McNally K, Khush GS, Paterson AH, Li ZK. Convergent evolution of perenniality in rice and sorghum. Proc Natl Acad Sci U S A. 2003;100:4050-4

12. Xu P, Dong L, Zhou J, Li J, Zhang Y, Hu F, Liu S, Wang Q, Deng W, Deng X. Identification and mapping of a novel blast resistance gene $P i_{57}(\mathrm{t})$ in Oryza longistaminata. Euphytica. 2015;205:95-102.

13. Ulitsky I, Bartel DP. LincRNAs: genomics, evolution, and mechanisms. Cell. 2013:154:26-46.

14. Chekanova JA. Long non-coding RNAs and their functions in plants. Curr Opin Plant Biol. 2015:27:207-16

15. Qin T, Zhao H, Cui P, Albesher N, Xiong L. A nucleus-localized long noncoding RNA enhances drought and salt stress tolerance. Plant Physiol. 2017; 175:1321-36.

16. Shumayla SS, Taneja M, Tyagi S, Singh K, Upadhyay SK. Survey of high throughput RNA-seq data reveals potential roles for IncRNAs during development and stress response in bread wheat. Front Plant Sci. 2017:8: 1019.

17. Wang Z, Liu Y, Li L, Li D, Zhang Q, Guo Y, Wang S, Zhong C, Huang H. Whole transcriptome sequencing of Pseudomonas syringae $\mathrm{pv}$ actinidiaeinfected kiwifruit plants reveals species-specific interaction between long non-coding RNA and coding genes. Sci Rep. 2017;7:4910.

18. Wang KC, Chang HY. Molecular mechanisms of long noncoding RNAs. Mol Cell. 2011:43:904-14.

19. Di C, Yuan J, Wu Y, Li J, Lin H, Hu L, Zhang T, Qi Y, Gerstein MB, Guo Y, Lu ZJ. Characterization of stress-responsive IncRNAs in Arabidopsis thaliana by integrating expression, epigenetic and structural features. Plant J. 2014;80: 848-61.

20. Wu HJ, Wang ZM, Wang M, Wang XJ. Widespread long noncoding RNAs as endogenous target mimics for microRNAs in plants. Plant Physiol. 2013;161: 1875-84.

21. Fan CY, Hao ZO, Yan JH, Li GL. Genome-wide identification and functional analysis of lincRNAs acting as miRNA targets or decoys in maize. BMC Genomics. 2015;16.

22. Datta R, Paul S. Long non-coding RNAs: fine-tuning the developmental responses in plants. J Biosci. 2019:44.

23. Campalans A, Kondorosi A, Crespi M. Enod40, a short open reading framecontaining mRNA, induces cytoplasmic localization of a nuclear RNA binding protein in Medicago truncatula. Plant Cell. 2004;16:1047-59.

24. Spitale RC, Tsai MC, Chang HY. RNA templating the epigenome long noncoding RNAs as molecular scaffolds. Epigenetics. 2011;6:539-43.

25. Swiezewski S, Liu FQ, Magusin A, Dean C. Cold-induced silencing by long antisense transcripts of an Arabidopsis polycomb target. Nature. 2009;462: 799-U122.

26. Heo JB, Sung S. Vernalization-mediated epigenetic silencing by a long intronic noncoding RNA. Science. 2011;331:76-9.

27. Franco-Zorrilla JM, Valli A, Todesco M, Mateos I, Puga MI, Rubio-Somoza I, Leyva A, Weigel D, Garcia JA. Paz-Ares J; target mimicry provides a new mechanism for regulation of microRNA activity. Nat Genet. 2007:39:1033-7. 
28. Li A, Liu D, Wu J, Zhao X, Hao M, Geng S, Yan J, Jiang X, Zhang L, Wu J, Yin $L$, Zhang $R$, Wu L, Zheng $Y$, Mao L. mRNA and small RNA transcriptomes reveal insights into dynamic homoeolog regulation of allopolyploid heterosis in nascent hexaploid wheat. Plant Cell. 2014;26:1878-900.

29. Shen YY, Zhao Q, Zou J, Wang WL, Gao Y, Meng JL, Wang JB. Characterization and expression patterns of small RNAs in synthesized Brassica hexaploids. Plant Mol Biol. 2014;85:287-99.

30. Vrbsky J, Akimcheva S, Watson JM, Turner TL, Daxinger L, Vyskot B, Aufsatz W, Riha K. siRNA-mediated methylation of Arabidopsis telomeres. PLoS Genet. 2010;6:e1000986.

31. Shao YL, Zhou HZ, Wu YR, Zhang H, Lin J, Jiang XY, He QJ, Zhu JS, Li Y, Yu $H$, Mao CZ. OsSPL3, an SBP-domain protein, regulates crown root development in rice. Plant Cell. 2019;31:1257-75

32. Lu ZF, Yu H, Xiong GS, Wang J, Jiao YQ, Liu GF, Jing YH, Meng XB, Hu XM, Qian Q, Fu XD, Wang YH, Li JY. Genome-wide binding analysis of the transcription activator IDEAL PLANT ARCHITECTURE1 reveals a complex network regulating rice plant ARCHITECTURE. Plant Cell. 2013;25:3743-59.

33. Iwamoto M, Kiyota S, Hanada A, Yamaguchi S, Takano M. The multiple contributions of phytochromes to the control of internode elongation in rice. Plant Physiol. 2011;157:1187-95.

34. Yoo MJ, Szadkowski E, Wendel JF. Homoeolog expression bias and expression level dominance in allopolyploid cotton. Heredity. 2013;110:171-80.

35. Zhang YC, Liao JY, Li ZY, Yu Y, Zhang JP, Li QF, Qu LH, Shu WS, Chen YQ. Genome-wide screening and functional analysis identify a large number of long noncoding RNAs involved in the sexual reproduction of rice. Genome Biol. 2014;15:512

36. Ding J, Lu Q, Ouyang Y, Mao H, Zhang P, Yao J, Xu C, Li X, Xiao J, Zhang Q. A long noncoding RNA regulates photoperiod-sensitive male sterility, an essential component of hybrid rice. Proc Natl Acad Sci U S A. 2012;109:2654-9.

37. Song JH, Cao JS, Wang CG. BCMF11, a novel non-coding RNA gene from Brassica campestris, is required for pollen development and male fertility. Plant Cell Rep. 2013:32:21-30.

38. Komiya R, Ohyanagi H, Niihama M, Watanabe T, Nakano M, Kurata N, Nonomura K. Rice germline-specific argonaute MEL1 protein binds to phasiRNAs generated from more than 700 lincRNAs. Plant J. 2014;78:385-97.

39. Liu H, Wang R, Mao B, Zhao B, Wang J. Identification of IncRNAs involved in rice ovule development and female gametophyte abortion by genome-wide screening and functional analysis. BMC Genomics. 2019;20:90.

40. Xin M, Wang Y, Yao Y, Song N, Hu Z, Qin D, Xie C, Peng H, Ni Z, Sun Q. Identification and characterization of wheat long non-protein coding RNAs responsive to powdery mildew infection and heat stress by using microarray analysis and SBS sequencing. BMC Plant Biol. 2011;11:61.

41. Jain $P$, Sharma $V$, Dubey $H$, Singh $P K$, Kapoor R, Kumari M, Singh J, Pawar DV, Bisht D, Solanke AU, Mondal TK, Sharma TR. Identification of long noncoding RNA in rice lines resistant to rice blast pathogen Maganaporthe oryzae. Bioinformation. 2017;13:249-55.

42. Jiang B, Lou Q, Wu Z, Zhang W, Wang D, Mbira KG, Weng Y, Chen J. Retrotransposon- and microsatellite sequence-associated genomic changes in early generations of a newly synthesized allotetraploid Cucumis $\times$ hytivus Chen \& Kirkbride. Plant Mol Biol. 2011;77:225-33.

43. Lu X, Chen X, Mu M, Wang J, Wang X, Wang D, Yin Z, Fan W, Wang S, Guo $L, Y e W$. Genome-wide analysis of long noncoding RNAs and their responses to drought stress in cotton (Gossypium hirsutum L.). PLoS One. 2016;11:e0156723.

44. Yuan J, Li J, Yang Y, Tan C, Zhu Y, Hu L, Qi Y, Lu ZJ. Stress-responsive regulation of long non-coding RNA polyadenylation in Oryza sativa. Plant J. 2018;93:814-27.

45. Itoh H, Tatsumi T, Sakamoto T, Otomo K, Toyomasu T, Kitano H, Ashikari M, Ichihara S, Matsuoka M. A rice semi-dwarf gene, Tan-Ginbozu (D35), encodes the gibberellin biosynthesis enzyme, ent-kaurene oxidase. Plant Mol Biol. 2004:54:533-47.

46. Itoh H, Shimada A, Ueguchi-Tanaka M, Kamiya N, Hasegawa Y, Ashikari M, Matsuoka M. Overexpression of a GRAS protein lacking the DELLA domain confers altered gibberellin responses in rice. Plant J. 2005;44:669-79.

47. Sasaki A, Itoh H, Gomi K, Ueguchi-Tanaka M, Ishiyama K, Kobayashi M, Jeong $\mathrm{DH}$, An G, Kitano H, Ashikari M, Matsuoka M. Accumulation of phosphorylated repressor for gibberellin signaling in an F-box mutant. Science. 2003;299:1896-8.

48. Wang Q, Zhang W, Yin Z, Wen CK. Rice CONSTITUTIVE TRIPLE-RESPONSE2 is involved in the ethylene-receptor signalling and regulation of various aspects of rice growth and development. J Exp Bot. 2013;64:4863-75.
49. Li G, Liang W, Zhang X, Ren H, Hu J, Bennett MJ, Zhang D. Rice actinbinding protein RMD is a key link in the auxin-actin regulatory loop that controls cell growth. Proc Natl Acad Sci U S A. 2014;111:10377-82.

50. Xu XW, Zhou XH, Wang RR, Peng WL, An Y, Chen LL. Functional analysis of long intergenic non-coding RNAs in phosphate-starved rice using competing endogenous RNA network. Sci Rep. 2016;6:20715.

51. Ge LF, Chao DY, Shi M, Zhu MZ, Gao JP, Lin HX. Overexpression of the trehalose-6-phosphate phosphatase gene OSTPP1 confers stress tolerance in rice and results in the activation of stress responsive genes. Planta. 2008; 228:191-201.

52. Manosalva PM, Bruce M, Leach JE. Rice 14-3-3 protein (GF14e) negatively affects cell death and disease resistance. Plant J. 2011;68:777-87.

53. Liu Q, Yang J, Zhang S, Zhao J, Feng A, Yang T, Wang X, Mao X, Dong J, Zhu X, Leung H, Leach JE, Liu B. OsGF14e positively regulates panicle blast resistance in rice. Biochem Biophys Res Commun. 2016:471:247-52.

54. Cheng H, Liu H, Deng Y, Xiao J, Li X, Wang S. The WRKY45-2 WRKY13 WRKY42 transcriptional regulatory cascade is required for rice resistance to fungal pathogen. Plant Physiol. 2015;167:1087-99.

55. Yang C, Li D, Mao D, Liu X, Ji C, Li X, Zhao X, Cheng Z, Chen C, Zhu L. Overexpression of microRNA319 impacts leaf morphogenesis and leads to enhanced cold tolerance in rice (Oryza sativa L.). Plant Cell Environ. 2013;36:2207-18.

56. Thirumurugan T, Ito Y, Kubo T, Serizawa A, Kurata N. Identification, characterization and interaction of HAP family genes in rice. Mol Gen Genomics. 2008;279:279-89.

57. Takano M, Inagaki N, Xie X, Kiyota S, Baba-Kasai A, Tanabata T, Shinomura T. Phytochromes are the sole photoreceptors for perceiving red/far-red light in rice. Proc Natl Acad Sci U S A. 2009;106:14705-10.

58. Li RQ, Li YR, Kristiansen K, Wang J. SOAP: short oligonucleotide alignment program. Bioinformatics. 2008;24:713-4.

59. Kim D, Landmead B, Salzberg SL. HISAT: a fast spliced aligner with low memory requirements. Nat Methods. 2015;12:357-U121.

60. Pertea M, Pertea GM, Antonescu CM, Chang T-C, Mendell JT, Salzberg SL. StringTie enables improved reconstruction of a transcriptome from RNA-seq reads. Nat Biotechnol. 2015;33:290-5.

61. Trapnell C, Williams BA, Pertea G, Mortazavi A, Kwan G, van Baren MJ, Salzberg SL, Wold BJ, Pachter L. Transcript assembly and quantification by RNA-Seq reveals unannotated transcripts and isoform switching during cell differentiation. Nat Biotechnol. 2010;28:511-U174.

62. Finn RD, Coggill P, Eberhardt RY, Eddy SR, Mistry J, Mitchell AL, Potter SC, Punta M, Qureshi M, Sangrador-Vegas A, Salazar GA, Tate J, Bateman A. The Pfam protein families database: towards a more sustainable future. Nucleic Acids Res. 2016:44:D279-85.

63. Kong L, Zhang Y, Ye ZQ, Liu XQ, Zhao SQ, Wei L, Gao G. CPC: assess the protein-coding potential of transcripts using sequence features and support vector machine. Nucleic Acids Res. 2007;35:W345-9.

64. Sun L, Luo H, Bu D, Zhao G, Yu K, Zhang C, Liu Y, Chen R, Zhao Y. Utilizing sequence intrinsic composition to classify protein-coding and long noncoding transcripts. Nucleic Acids Res. 2013:41.

65. Langmead B, Salzberg SL. Fast gapped-read alignment with bowtie 2. Nat Methods. 2012:9:357-U354.

66. Li B, Dewey CN. RSEM: accurate transcript quantification from RNA-Seq data with or without a reference genome. BMC Bioinformatics. 2011;12:323.

67. Wang L, Feng Z, Wang X, Wang X, Zhang X. DEGseq: an R package for identifying differentially expressed genes from RNA-seq data. Bioinformatics. 2010;26:136-8.

68. Knauss $J$, Sun T. Regulatory mechanisms of long noncoding RNAs in vertebrate central nervous system development and function. Neuroscience. 2013;235:200-14.

69. Kornienko AE, Guenzl PM, Barlow DP, Pauler FM. Gene regulation by the act of long non-coding RNA transcription. BMC Biol. 2013;11:59.

70. Kozomara A, Birgaoanu M, Griffiths-Jones S. miRBase: from microRNA sequences to function. Nucleic Acids Res. 2019;47:D155-62.

71. Kubo T, Fujita M, Takahashi H, Nakazono M, Tsutsumi N, Kurata N. Transcriptome analysis of developing ovules in rice isolated by laser microdissection. Plant Cell Physiol. 2013;54:750-65.

\section{Publisher's Note}

Springer Nature remains neutral with regard to jurisdictional claims in published maps and institutional affiliations. 